\title{
Un panorama del efecto Foucault en contabilidad y gestión pública temáticas, autores y retos de investigación desde la gubernamentalidad
}

\author{
An Overview of Foucault's Effect in the Public Management and Accounting. Themes, Authors, and Research \\ Challenges from the Governability \\ Panorama do efeito Foucault na contabilidade e gestão pública Temáticas, autores e desafios de pesquisa da \\ governamentalidade
}

Carmen Alejandra Ocampo-Salazar

Universidad Eafit, Colombia

DOI: https://doi.org/10.11144/Javeriana.cc19-47.pefc Redalyc: http://www.redalyc.org/articulo.oa?

cocampo2@eafit.edu.co

id $=151556994003$

ORCID: http://orcid.org/0000-0003-4417-2683

Fecha de recepción: 06 Octubre 2017

Fecha de aprobación: 13 Marzo 2018

\section{Resumen:}

En este documento se presenta un panorama del efecto Foucault en contabilidad y gestión pública, con el fin de evidenciar temáticas, autores y vacíos de investigación desde los estudios en gubernamentalidad. La unidad de análisis fueron setenta textos seleccionados en una revisión sistemática de artículos publicados entre 2012 y 2016, en cinco de las principales revistas internacionales relacionadas con las disciplinas analizadas. Para la recolección de los datos y la codificación de la información se utilizó una matriz de Excel y el software de investigación cualitativa Atlas.ti, versión 7.5.16. La comprensión de la información se complementó con un método de análisis de frecuencia y un análisis de red. Dentro de los resultados se destacan cuatro ejes temáticos: la gestión calculada, el gobierno a distancia, la modernización del gobierno y la contabilidad de la ciudad. Además, los cinco autores que más han publicado en el tema: Dean Neu, Cameron Graham, Eric Pezet, Ingrid Jeacle y Peter Miller, así como las redes de cooperación entre países, dentro de los cuales se evidencia a Reino Unido y Canadá. También se identifica que el principal reto de investigación está en entender la contabilidad de las ciudades de las llamadas economías emergentes. Así, este artículo hace contribuciones importantes para la contaduría y la administración, en especial, se establecen herramientas teóricas para que los investigadores estudien la contabilidad y su incidencia en la gestión de las ciudades, desde la perspectiva de la gubernamentalidad, en aras de avanzar en este tópico que requiere de discusión en Latinoamérica para repensar las incidencias del cálculo contable y la gestión del ciudadano.

Palabras clave: Foucault, contabilidad, gestión pública, gubernamentalidad, investigación contable.

\section{Abstract:}

This paper presents an overview of the Foucault's effect on the public management and accounting in order to disclose the themes, authors and research voids based on the studies of governability. The corpus consisted of seventy texts selected through a systematic review of articles published between 2012 and 2016 in five of the main international journals related to the disciplines under study. An Excel matrix and the qualitative research software Atlas.ti (version 7.5.16) were used in the data collection and coding of the information. The understanding of the information was complemented with a frequency analysis method and a network analysis. Four theme lines stand out in the work outcomes: the calculated management; the distance government; the modernization of the government; and the city accounting. In addition, the five authors with most publications on this subject-matter are Dean Neu, Cameron Graham, Eric Pezet, Ingrid Jeacle and Peter Miller. Regarding the cooperation networks between countries the one between United Kingdom and Canada is highlighted. The main research challenge has been identified as how to understand the city accounting in those named emerging economies. Thus, this article make some important contributions to the accountancy and administration, especially by establishing the theoretical tools for the researchers to study accounting and how it influence the city management under a governability perspective. The goal is to make progress on this topic, which needs to be discussed in Latin America so as to rethink the impacts of both the accounting calculation and citizen's management.

Keywords: Foucault, accounting, public management, governability, accounting research.

\section{Resumo:}

Neste documento apresenta-se um panorama do efeito Foucault na contabilidade e gestão pública a fim de destacar temáticas, autores e lacunas de pesquisa desde os estudos em governamentalidade. A unidade de análise foi setenta textos selecionados em uma 
revisão sistemática de artigos publicados entre 2012 e 2016, em cinco das principais revistas internacionais relacionadas com as disciplinas analisadas. Para a coleta dos dados e a codificação da informação foi usada uma matriz de Excel e o software de pesquisa qualitativa Atlas.ti, versão 7.5.16. A compreensão da informação foi complementada com um método de análise de frequência e uma análise de rede. Dentro dos resultados destacam quatro eixos temáticos: gestão calculada, governo a distancia, modernização do governo e contabilidade da cidade. Além disso, os cinco autores que mais publicaram sobre o tema: Dean Neu, Cameron Graham, Eric Pezet, Ingrid Jeacle e Peter Miller, bem como as redes de cooperação entre países, dentro dos quais se evidencia Reino Unido e Canadá. Mesmo identificou-se que o principal desafio de pesquisa está em entender a contabilidade das cidades das chamadas de economias emergentes. Assim, este artigo faz contribuições importantes para a contabilidade e a administração, em especial, estabelecem-se ferramentas teóricas para os pesquisadores estudarem a contabilidade e sua incidência na gestão das cidades, desde a perspectiva da governamentalidade, a fim de avançar neste tópico que requer de discussão em Latino América para repensar as incidências do cálculo contábil e a gestão do cidadão.

Palavras-chave: Foucault, contabilidade, gestão pública, governamentalidade, pesquisa contábil.

\section{Introducción}

La investigación en contabilidad y gestión pública ha tomado como referencia los estudios de gubernamentalidad de Michel Foucault, para comprender que la contabilidad es una tecnología política y social que incide en el gobierno de la población (Burchell, Clubb, Hopwood, Hughes y Nahapiet, 1980; Miller y O’leary, 1987; Armstrong, 1994; Napier, 2006, y Lambert y Pezet, 2012). Bajo el enfoque foucaultiano, los estudios sociales de la contabilidad reconocen que ella no refleja neutralmente la realidad en las organizaciones, sino que constituye un conjunto de prácticas que afecta al mundo en el que vivimos, el tipo de realidad social que habitamos, la manera como entendemos la empresa y las personas, la forma como gestionamos y organizamos actividades y procesos, y en la que administramos la vida individual y de los demás (Miller, 1994). En esta perspectiva, la contabilidad, desde sus tecnologías y programas, no actúa directamente sobre las personas, sino sobre sus reglas de juego y campos de elección.

A finales de la década de los años setenta, algunos académicos inspirados en el trabajo de Foucault difundieron nuevas coordenadas para orientar la investigación contable y de gestión desde los ambientes en los que ellas operan. Así, el profesor inglés Anthony Hopwood, al fundar la revista Accounting, Organizations and Society, en 1976, abrió una agenda investigativa de problemas relacionados con los contextos que afectan o son afectados por la contabilidad. Trabajos iniciales como los de Burchell et al. (1980) implicaron un giro epistemológico y metodológico de la investigación contable, al reconocer que el funcionamiento de las instituciones sociales, el cambio y el contexto social eran elementos significativos que se debían estudiar en su relación con lo contable. En este sentido, Napier (2006) y Walker (2016) en la revisión de las publicaciones del citado journal, reconocieron que estos estudios se distancian de la visión dominante en contabilidad, difundida principalmente en Estados Unidos. Una de sus principales diferencias está en la comprensión de la contabilidad como una práctica social e institucional clave en la configuración de la sociedad.

A partir de 1990, los miembros de la red Historia del presente (Miller, Rose, Gordon, Burchell, Dean y otros), creada en Londres en 1989, marcaron una tendencia más explícita para los estudios foucaultianos. En esa época, los ingleses Miller y Rose (1990) describieron algunos modos de gobernar la vida económica, mientras florecieron estudios que articularon el concepto de tecnología de gobierno. Entre ellos, el libro The Foucault Effect: Studies in Governmentality, editado por Burchell, Gordon y Miller en 1991, en el cual se amplió de forma significativa la atención sobre esta perspectiva. Igualmente, el texto Accounting as Social and Institutional Practice, de Hopwood y Miller, publicado en 1994, se convirtió en un referente para comprender la contabilidad como tecnología calculadora. También, el libro Governing the Present: Administering Economic, Social and Personal Life, de Rose y Miller (2008), divulgó una serie de artículos de la década de los años noventa, clave para estudiar las prácticas de cálculo y el gobierno de sí. En conjunto con esta publicación, en 2008, la University of Edinburgh realizó el seminario Contabilidad para las ciudades, que contó con un número especial en la revista Accounting, Auditing \& Accountability Journal; en ese evento 
participaron los autores del citado texto, quienes mostraron que el estudio de la contabilidad y de la gestión de las ciudades no había sido abordado de forma significativa por los contables. Y desde este evento se evidencian los retos de investigación para este trabajo, dado el interés personal por estudiar la contabilidad en el ámbito de la ciudad y sus implicaciones en la gestión del ciudadano.

En consecuencia, el presente artículo tiene como objetivo analizar el efecto Foucault en la investigación contable y de gestión pública de los últimos quince años (2002-2016), en aras de reconocer temáticas, autores, problematizaciones y rutas de trabajo en contabilidad y administración. A nivel metodológico, se identificaron revistas que han publicado textos paradigmáticos de las corrientes heterodoxas en contabilidad, se destacan: Accounting, Organizations and Society (Imperial College London), Financial Accountability \& Management (University of Edinburgh), Accounting, Auditing \& Accountability Journal (University of Sheffield) y Critical Perspectives on Accounting (The University of York-University of Strathclyde), con sedes en Reino Unido y, la última, en Canadá. En estas publicaciones se observa el uso de elementos de la caja de herramienta de Foucault y de sus intérpretes, quienes se apropiaron del análisis foucaultiano y lo difundieron por el mundo.

Para desarrollar el objetivo propuesto en este trabajo, el texto se divide en cuatro secciones adicionales a esta parte introductoria. En la primera se presentan los referentes teóricos a partir de los cuales se revisaron los artículos objeto de análisis. En la segunda se expone la metodología utilizada en el análisis de los artículos. En la tercera se explican los resultados de este estudio. Por último, se plantean algunas discusiones y conclusiones respecto a los hallazgos, además de algunas limitaciones del trabajo realizado.

\section{Referentes teóricos desde los estudios en gubernamentalidad}

El tema de la gubernamentalidad tuvo un lugar central en los análisis de Foucault entre 1976 y 1984, como un tipo de poder que ha permitido conducir la conducta de la población mediante la conquista de la libertad, la vida, el alma y con ello la decisión moral de las personas. Desde las reflexiones foucaultianas y de autores como Donzelot, Ewald, Hacking y Procacci, los estudios en gubernamentalidad se reconocen como una zona de investigación inacabada (Gordon, 1991, p. 2), un punto de encuentro que permite hacer visible las diferentes formas en las que una actividad o arte denominado gobierno ha sido pensable y practicable (Burchell et al., 1991). En este sentido, este apartado explica la categoría de gubernamentalidad como un poder-gobierno y su relación con los estudios sociales para comprender la contabilidad y la gestión en el ámbito estatal.

\section{Gubernamentalidad, un poder-gobierno}

En 1957, Roland Barthes (1999) acunó "un neologismo bárbaro pero inevitable: la gubernamentalidad, el gobierno concebido por la gran prensa como esencia de la eficacia" (p. 121). Este término se convirtió en uno de los conceptos clave en el léxico filosófico e histórico de los últimos trabajos de Foucault (McKinlay y Pezet, 2010), para explicar que la conducta es una de las mejores guías que permite entender las relaciones de poder como una cuestión más de gobierno que de confrontación entre adversarios (Foucault, 2001). La concepción foucaultiana de gobierno trazó dos ejes básicos: "el gobierno como relación entre sujetos y el gobierno como relación consigo mismo" (Castro, 2007, p. 11). El primero, desarrollado en el curso Seguridad, territorio, población, de 1978, presentó un gobierno que tiene por objeto la conducta de otro individuo o grupo, mientras que el segundo, planteado en la conferencia sobre Tecnología del yo, de 1982, hizo referencia al autogobierno.

En relación con el primer eje de gubernamentalidad, Foucault (2011) entendió, entre otros aspectos: 
el conjunto constituido por las instituciones, los procedimientos, análisis y reflexiones, los cálculos y las tácticas que permiten ejercer esa forma bien específica, aunque muy compleja, de poder que tiene por blanco principal la población, por forma mayor de saber la economía política y por instrumento técnico esencial los dispositivos de seguridad. (p. 136)

En esta concepción, la población se identifica como un problema clave, una realidad dinámica abordada por saberes expertos como la economía política y técnicas como la estadística (Castro-Gómez, 2010). La población no constituye entonces un dato básico, sino un conjunto de procesos que dependen del entorno material y social (Foucault, 2011).

En concordancia con el segundo eje de gubernamentalidad -el gobierno de sí-, Foucault (1994) planteó que "el sí mismo por el que uno se preocupa ha dejado de ser un elemento entre otros, ha dejado de ser un enlace, una bisagra, un elemento de transición tendiente a otra cosa que sería la ciudad o los otros. El sí mismo se convierte en el objetivo definitivo y único de la preocupación por uno mismo" (pp. 67-68), es su propio fin en la actividad que se ejerce sobre sí. En lo anterior hay un elemento significativo para la gubernamentalidad denominado "tecnologías de gobierno", que constituyen una dimensión estratégica de las prácticas que posibilitan conducir la conducta de los sujetos (sujeción) y permiten, a la vez, que los sujetos dirijan autónomamente su propia conducta (subjetivación).

Así, las tecnologías de gobierno se comprenden como aquellas que hacen posible que los sujetos establezcan relaciones con los demás y consigo mismos, en aras de dirigir la conducta desde la aprobación del otro, desde sus condiciones de aceptabilidad. Por ende, "el gobierno sobre la conducta nunca es obligado, nunca se hace en contra de la propia voluntad” (Castro-Gómez, 2010), sino mediante una acción a distancia; Foucault (2011) lo deja entrever al indicar que el poder de gobierno influye "sobre cosas aparentemente alejadas de la población, pero que, según hacen saber el cálculo, el análisis y la reflexión, pueden actuar en concreto sobre ella” (p. 95). Allí la gubernamentalidad implica una acción a distancia que no se ejerce sobre los jugadores, sino sobre sus reglas de juego (Foucault, 2008); una racionalidad que en las prácticas de subjetivación propone a la población formas de gobierno sobre sí mismos, con mecanismos indirectos que buscan gobernar la vida de los hombres, conducir su conducta política y moral hacia los objetivos del modelo neoliberal.

Foucault (2008) caracterizó el neoliberalismo como el conjunto de intervenciones en el ambiente de los marcos de acción de los sujetos económicos. En sus cursos de 1978 y 1979, explicó su versión contemporánea desde dos escuelas neoliberales: el ordoliberalismo alemán y el neoliberalismo norteamericano. La primera plantea la formación de la sociedad desde el modelo de empresa y la intervención indirecta mediante reglas de juego para los ciudadanos; mientras que la segunda implica la creación de un ambiente de riesgo, al considerar que la inseguridad estimula la competitividad y el autogobierno de las personas. Desde allí planteó que la era de la vigilancia llevada a cabo en el Estado benefactor quedó atrás con el nuevo arte neoliberal de gobierno que opera creando un medio ambiente para que los gobernados puedan actuar con libertad (Castro-Gómez, 2010). Por ende, en su analítica de la gubernamentalidad reconoció un nuevo régimen de verdad: la economía política, un saber mediante el cual la gestión de la población cambia de significación y de método; la finalidad del Estado, acorde con el auge neoliberal, ahora es dirigir y adaptar las sociedades a las exigencias de la competencia mundial y de las finanzas globales (Laval y Dardot, 2013), una valoración de la población por su contribución a la competitividad.

En esta perspectiva, Laval y Dardot (2013) plantearon en su texto La nueva razón del mundo: ensayo sobre la sociedad neoliberal, una genealogía de la representación utilitarista del neoliberalismo, una racionalidad política que tiende a estructurar y organizar la acción de los gobernantes y la conducta de los propios gobernados, con la competencia como norma de tal conducta y la empresa como modelo de subjetivación. Por lo tanto, los citados autores plantean que el neoliberalismo define cierta forma de vida en las sociedades occidentales y en aquellas que le siguen en el camino a la modernidad:

Esta norma obliga a cada uno a vivir en un universo de competición generalizada, impone tanto a los asalariados como a las poblaciones que entren en una lucha económica unos con otros, sujeta las relaciones sociales al mercado, empuja a justificar desigualdades cada vez mayores, transforma también al individuo, que en adelante es llamado a concebirse y a conducirse 
como una empresa. Desde hace más de treinta años, esta norma de existencia preside las políticas públicas, rige las relaciones económicas mundiales, remodela la subjetividad. (p. 14)

A partir de lo expuesto se reconoce un cambio sustantivo en la racionalidad del gobierno, del tipo de tecnologías y programas mediante las cuales el gobierno es realizado. Allí las tecnologías aluden a esa dimensión estratégica de las prácticas, mientras que los programas son prácticas discursivas que presuponen que lo real es programable y, por ende, puede ser sometido al dominio de reglas, normas, procesos e intervenciones calculadas (Miller y Rose, 2008). Desde los estudios en gubernamentalidad se han realizado una serie de investigaciones sobre las tecnologías y programas de gobierno, dos términos gemelos que han permitido delinear una agenda de investigación con reflexiones de quienes han abordado de forma heterodoxa las prácticas contables y de gestión, prácticas en el sentido de una línea de intersección entre el sujeto, aquello que se constituye como verdad, y la historia.

\section{Contabilidad y gestión en el ámbito estatal}

Durante la década de los años setenta se evidenció el agotamiento del modelo industrial en Occidente y una mutación histórica del capitalismo industrial hacia un capitalismo mundializado y financiero (Boltanski y Chiapello, 2002). A partir de este cambio fue más evidente el paso de las sociedades disciplinarias a las sociedades de control; la empresa remplaza a la fábrica y constituye la forma hegemónica de la interacción social (Deleuze, 1999), donde adquiere sentido el management (Legendre, 2008; Aubert y De Gaudelag, 1993) como un modo de gestión susceptible de generalizarse en todos los ámbitos de la sociedad. Esta importante mutación de la sociedad capitalista ha permitido entender algunas de las transformaciones del Estado en Occidente: el paso de un Estado benefactor, propio de la sociedad disciplinaria, a un Estado neoliberal, que en el ámbito de la sociedad de control ha insertado la lógica managerial al gobierno de lo público.

Entre algunas de las condiciones de posibilidad histórica que se relacionan con este cambio de racionalidad política se resalta la crisis energética de 1973, que reveló una vulnerabilidad y reducción de la capacidad de acción del Estado (Chevallier, 2011) ante problemáticas como el desempleo y la inflación. Aunado a ello, condiciones como la crisis fiscal del Estado benefactor (O'Connor, 2009), su falta de legitimidad (Cabrero, 2011) y las contradicciones que se le atribuyeron al considerarlo costoso, ineficaz, ineficiente, represivo e ideológico (Offe, 1991), posibilitaron que los países desarrollados reinventaran la gestión pública con la inserción de prácticas y discursos del sector empresarial.

Así, la década de los años ochenta evidencia la apertura de una línea de pensamiento denominada Nueva Gestión Pública (NGP) (Hood, 1991; 1995) o gobierno empresarial (Osborne y Gaebler, 1994), que surgió en los contextos inglés y norteamericano con los gobiernos de Margaret Thatcher (1979-1990), en Inglaterra, y Ronald Reagan (1981-1989), en Estados Unidos. Esta doctrina, que ha sido dominante en los últimos años (incluso en los países subdesarrollados), constituye un modus operandi que generaliza la forma-empresa sobre la forma-Estado (Du Gay, 2003; 2012). Allí, la inserción de la lógica gerencial en la administración pública ha implicado el traslado de prácticas contables empresariales al ámbito estatal. Estas prácticas contables, a diferencia de lo que pasaba en el régimen fabril, contienen un alto componente económico y financiero, ya que en el contexto del capitalismo mundializado y financiero la contabilidad fue redefinida para traer a la luz instrumentos financieros como inversiones, riesgos, dividendos, préstamos y capital intelectual, entre otros.

En esta hegemonía de las finanzas y de la forma-empresa (financiera), la contabilidad de caja propia del gobierno en el Estado benefactor se concibió como un espejo imperfecto de la realidad estatal, que no permitía calcular cuánto debe el ente público, ni cuánto tiene, sino solo valorar los aportes cuando se recibe (ingreso) o paga (gasto) el efectivo. Esta transición del sistema contable de caja a una base acumulativa (o devengo) ha revelado un cambio de episteme en pro de una mayor eficiencia gerencial del gobierno, a la luz de los 
criterios de verdad que se constituyen desde la economía financiera. Por ende, el traslado de prácticas contables empresariales al ámbito público permite observar que el Estado se ha tornado en un agente dependiente de los mercados financieros, auspiciado por grandes operadores internacionales como el Banco Mundial, el Fondo Monetario Internacional y la Organización para la Cooperación y el Desarrollo Económico, entidades que han incentivado la transformación del lenguaje del ente público en nombre de la competitividad.

En este sentido, las reformas de la contabilidad pública (particularmente por partida doble e International Public Sector Accounting Standards, Ipsas) han buscado mejorar los discursos de transparencia, calidad y comparabilidad de la información financiera presentada por las entidades públicas en el mundo contemporáneo (Cîrstea, 2014). Este tipo de contabilidad, que implica un cálculo del espacio y el tiempo (histórico), selecciona aquello que interesa hacer evidente en el sistema capitalista y excluye lo que no le es útil informar. Así, las reglas de juego que han variado con las reformas de la contabilidad pública, se transforman para mejorar la revelación, la toma de decisiones y el control social con criterios propios del mercado, entre ellos: espíritu empresarial, endeudamiento, riesgo, innovación, transparencia, eficiencia y eficacia, entre otros.

Con base en lo anterior, se destaca que uno de los aspectos clave de estos cambios en la gestión pública ha sido el énfasis en la relación acreedor-deudor que permitió la nueva contabilidad, el capital como gran acreedor convierte a la población en deudora (sin distinción entre trabajadores, desempleados, pobres y ricos); "esta relación se ha pensado y programado como el núcleo estratégico de las políticas neoliberales" (Lazzarato, 2013, p. 30), todos somos responsable de la deuda pública en el Estado endeudado, en el cual el crédito se convirtió en uno de los principales medios para satisfacer las necesidades de la sociedad (Hardt y Negri, 2012). Desde allí se observa que la contabilidad ha participado de la transformación de categorías discursivas y prácticas de gobierno que trascienden e impactan la constitución de la subjetividad (por ejemplo: sujetos endeudados), pues la calculabilidad de las transacciones que representa la contabilidad ha implicado una verdad, un terreno común para la acción política, una confianza en la medición, en los números, en las cifras y en los rankings.

En el contexto histórico de este cambio se evidencia que la contabilidad puesta en otro escenario, en otra forma organizacional (el Estado), se constituye como en una tecnología de gobierno neoliberal que moviliza una variedad de políticas públicas y programas de gobierno que inciden en la gestión de la población. Esta analítica implica una concepción concreta (histórica y situada) de la contabilidad como una práctica social e institucional en la organización, una compleja interacción entre el cálculo y la gestión de la vida social y organizacional (Burchell et al., 1980; Hopwood, 1992; Miller, 1994). En el margen de la contingencia histórica, estas prácticas contables se entienden como lugares en los cuales se instauran ciertas reglas de juego, que aunadas al discurso apropian una serie de instancias del lenguaje económico-financiero e instituyen un espacio de emergencia de los conceptos y de las relaciones de los sujetos con aquello que se define como verdad.

A partir de este posicionamiento teórico se revisó la literatura objeto de análisis en este artículo, con el fin de exponer un panorama actual del efecto que ha tenido Michel Foucault en los estudios contables y de gestión pública desde la perspectiva de la gubernamentalidad.

\section{Metodología del efecto Foucault}

El efecto ha sido considerado como un sentido, no solo causal, sino un efecto en el sentido óptico, sonoro o, mejor aún, de superficie, de posición, de lenguaje (Deleuze, 1994). El nombre propio -de Foucault- ha sido considerado como un símbolo, en la medida en que remite a un efecto de este tipo, por lo tanto, se habla del "efecto Foucault" para hacer visible, a través de una perspectiva particular en la Historia del presente, las diferentes formas en las que la gubernamentalidad se ha hecho pensable y practicable (Burchell et al., 1991) en la gestión y en la contabilidad pública. Si bien la cuestión de la gubernamentalidad no se vincula solo con los análisis de estas prácticas en lo público, se aborda este sector porque permite evidenciar los límites 
y cambios que genera el traslado de la lógica gerencial al ámbito estatal. Aunado a ello está la intención de atender el llamado realizado en el seminario de "Contabilidad sobre las ciudades", al que se aludió en la parte introductoria de este texto.

En consecuencia, se identificaron algunas revisiones de literatura que estudian el efecto Foucault en la investigación en contabilidad y gestión, no obstante, estos trabajos no enfatizaron propiamente en el ámbito estatal, ni en la categoría de gubernamentalidad. Así, Armstrong (1994) y Rivera (2012) detallan un análisis de artículos contables y de gestión en perspectiva foucaultiana. También, Chiapello y Baker (2011) presentan la influencia de la teoría francesa en el lenguaje académico de la contabilidad y destacan allí las referencias realizadas por Michel Foucault. Por su parte, Chiapello (2016) muestra la ampliación de los enfoques del neoliberalismo -entre ellos la gubernamentalidad-y las limitaciones para la investigación crítica en contabilidad; sin embargo, no articula el NPM en su exposición y reflexión. Otras recensiones se centraron en mostrar la evolución de los estudios contables y de gestión en el sector público, sin tipificar la incidencia de Foucault en tales investigaciones. En esta línea se relacionan los textos de Broadbent y Guthrie (1992; 2008), Brusca (2010), Helden y Uddin ${ }^{[1]}$ (2016), y Anessi-Pessina, Barbera, Sicilia y Steccolini (2016).

En aras de aportar a estas recensiones se realizó un análisis específico del efecto Foucault en las disciplinas objeto de este estudio (contabilidad y gestión pública), en los últimos quince años (2002-2016). Para este propósito se consideraron las revistas científicas, ya que estas constituyen una pieza clave de difusión de resultados de investigación, debido a su publicación periódica, además de que posibilitan reconocer reflexiones de punta y tendencias de estudio (Viana de Souza, Corréa da Silva \& Oliveira, 2013). En este trabajo se adaptaron algunos elementos del método de revisión estructurada de literatura propuesto por Massaro, Dumay y Guthrie (2016). Entre ellos, los criterios de selección de las revistas, tales como la perspectiva del journal, su trayectoria en contabilidad y administración, y su reconocimiento en los estados de la cuestión referenciados anteriormente. Así se optó por la consulta y revisión de las siguientes revistas: Accounting, Organizations and Society (AOS), Accounting, Auditing \& Accountability Journal (AAAJ), Critical Perspectives on Accounting (CPA), Management Accounting Research (MAR) y Foucault Studies (FS).

Para la recolección de la información en estas revistas se elaboró una matriz analítica con los siguientes datos: título del artículo, nombre de autor(es), dependencia de adscripción y país, resumen, palabras clave, temáticas abordadas y tipo de investigación. En este proceso se identificaron setenta artículos (objeto de estudio) que fueron analizados con el software Atlas.ti, versión 7.5.16, con base en los siguientes interrogantes: ¿Qué problematizaciones han sido abordadas en la investigación contable y de gestión pública desde la categoría de gubernamentalidad? ¿En qué ámbitos del sector público se han centrado este tipo de investigaciones? ¿Cuáles son los principales autores en esta perspectiva de investigación y sus universidades de adscripción? ¿Qué redes de cooperación se evidencian en el ámbito de la gestión pública y el pensamiento contable heterodoxo? ¿Qué vacíos se identifican en los textos revisados y cuáles son los retos que proponen a nivel investigativo?

En el análisis de estas preguntas, pese al carácter descriptivo de la revisión de literatura, se devela un posicionamiento crítico respecto a la NGP (específicamente con el traslado de prácticas contables del ámbito privado al sector público), una problemática emergente que ha implicado tramas de poder que constituyen al Estado neoliberal. En particular, este posicionamiento en la codificación de los datos permitió dar sentido a la construcción de las categorías abordadas en los resultados de este estudio: 1) problematizaciones y ámbitos de gobierno; 2) autores y redes de cooperación desde el pensamiento contable heterodoxo, y 3) vacíos y retos de investigación. 


\section{Resultados}

Este apartado presenta en detalle el análisis de las categorías construidas en la revisión de la literatura; por consiguiente, se exponen las problematizaciones y ámbitos de gobierno desde cuatro ejes que relacionan la gestión y la contabilidad en el sector público. Seguidamente, se especifican los principales autores y las redes de cooperación que se evidencian en los estudios contables heterodoxos y el papel del pensamiento inglés en este proceso. Finalmente, se evalúan los vacíos de investigación planteados por los autores en los artículos relevados, en aras de mostrar posibilidades de investigación futura.

\section{Problematizaciones y ámbitos de gobierno}

La categoría problematización alude al:

Conjunto de prácticas discursivas y no discursivas que hacen que algo entre en el juego de lo verdadero y de lo falso y lo constituye como objeto de pensamiento (bien sea en la forma de reflexión moral, del conocimiento científico, del análisis político...) (Foucault, 1999, p. 371).

En esta perspectiva, el trabajo busca recobrar el sentido de los problemas mediante la tematización de los textos objeto de estudio, en relación con el contexto social e institucional (ámbito de gobierno) en el que la contabilidad pública se ha tornado en una práctica que determina "lo verdadero" en la gestión estatal. Como resultado, se construyeron cuatro ejes temáticos -sobre otros posibles (incluso tecnologías y programas que son transversales)-, porque estos permiten resaltar discusiones emergentes relacionadas con la inserción de la lógica calculadora en la administración estatal. El detalle aparece en la Figura 1 con el número de investigaciones clasificadas en cada eje:

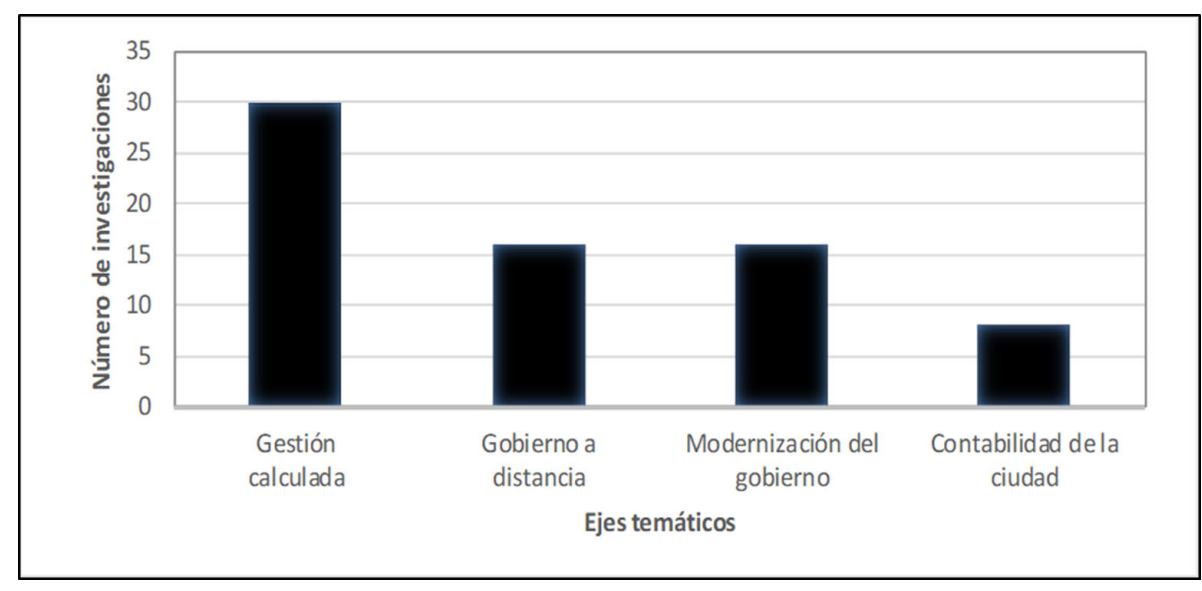

FIGURA 1.

Número de investigaciones por eje temático.

Fuente: elaboración propia.

En el eje temático de la gestión calculada se identificaron 31 trabajos, cuyos autores definen que, en general, la contabilidad pública constituye una tecnología de cálculo intrínseca a la actividad de la gestión gubernamental. Una especie de axioma en el que lo medible es lo gestionable (Vaivio, 2006; Tregidga, 2013; Lukka y Vinnari, 2014), ya que las métricas de la contabilidad en la administración permiten guiar y controlar la conducta de las personas (Lambert, 2010), transformar las prácticas organizacionales (Jones, 2010) y constituir contextos sociales y culturales en los que ella opera (Jeacle, 2012; McKinlay, Carter, Pezet y Clegg, 2010). Así, las cifras contables se han movilizado por una variedad de políticas públicas que intervienen la vida de las personas, configuran sujetos, dominios y acciones que supuestamente se tornan objetivas y comparables 
(Mennicken y Miller, 2012). En este sentido, los profesores Watkins y Arrington (2007) plantean que en el marco de la NPM, las diversas funciones públicas y los léxicos de evaluación se traducen en un discurso dominado por el cálculo económico, la gestión eficiente y el buen gobierno empresarial.

Entre otras temáticas y ámbitos estatales se analizaron las políticas de inmigración de economías avanzadas, en tanto espacios calculables y auditables (Lehman, Annisette y Agyemang, 2016), las clasificaciones contables y los rankings como formas de regulación del gobierno global (Mehrpouya y Samiolo, 2016), los espacios dominantes del crédito (Jeacle, 2012), las relaciones entre contabilidad, escritura y dinero (Ezzamel y Hoskin, 2002), el cálculo actuarial de pensiones y la depreciación humana en Estados Unidos y Chile (Himick, 2009; 2015; 2016), además de la hibridación contable para hacer visible y gobernable las incertidumbres asociadas con la sostenibilidad ambiental (Thomson, Grubnic y Georgakopoulos, 2014). También, en la literatura aparecen problemas con la operatividad de los riesgos como un espacio calculable que crea una falsa sensación de seguridad (Mahama y Yu Ming, 2009; Huber y Scheytt, 2013; Jordan, Mitterhofer y Jørgensen, 2016), los cálculos contables e intervenciones en torno a la hambruna irlandesa entre 1846-1847 (O’Regan, 2010), al gobierno de los pobres en Italia durante los siglos XVI-XVII (Servalli, 2013), a la gestión de la corrupción de la élites dentro de los juegos de verdad (Hoskin, 2015) y a los beneficios fiscales en el gobierno inglés (Edgley, 2010).

La gestión calculada se expande en las sociedades de control con mediciones de la contabilidad que permiten ordenar, clasificar y adoptar nuevas prácticas para el gobierno (Martínez, 2011). El dominio de lo económico y lo financiero es un fundamento de este cálculo contable, como una forma de conocer y gestionar las organizaciones. Entre estos trabajos se observa un estudio sobre las "almas humanas" desde los cálculos contables en la supervisión educativa (Walker, 2010) y las tecnologías de la contabilidad vinculadas al cálculo de sí mismos, si toma la forma de empresario (Giraudeau, 2012), de jubilado (Graham, 2010; 2012), de gerente (Lambert y Pezet, 2012) o de sujeto de la moda (Jeacle, 2012). Allí la contabilidad (sus prácticas) permite visibilizar la organización desde el lenguaje financiero, que incide en la elaboración de los programas de gobierno y en las relaciones sociales que forma y en aquellas que busca administrar.

Ahora bien, en el eje temático del gobierno a distancia se identificaron 17 investigaciones. Este eje relaciona de forma explícita la relevancia de la contabilidad dentro del contexto en el cual se aplica. Aunque la distancia no alude necesariamente a un espacio físico entre dos puntos, como en la geometría euclidiana, sí denota aquello que separa un contexto del otro. En este sentido, la distancia implica la capacidad de influir en muchos contextos, de percibir el espacio social como un proceso, una construcción, un reflejo de las condiciones políticas, culturales, económicas y geográficas del mundo contemporáneo (Corvellec, Ek, Zapata y Zapata, 2016). Allí las prácticas contables han jugado un papel relevante en la administración pública, pues han permitido extender el control y la autoridad no solo en el ambiente interno sino externo (Asdal, 2011). Por ende, han fomentado la acción a distancia no solo desde el nivel jerárquico (Carmona, Ezzamel y Gutiérrez, 2002; Neu y Graham, 2004), sino el discursivo e institucional (Neu, 2006), cultural (Neu y Graham, 2004; 2006; Preston, 2006), el moral (Baker, 2010; Bigoni y Funnell, 2015), el de endeudamiento (Neu, Ocampo, Graham y Heincke, 2006), el de visibilidad social (Neu, Everett y Rahaman, 2015) y el de conflicto (Thomson, Dey y Russell, 2015).

La contabilidad es constitutiva del espacio y del tiempo, es decir, es una práctica social e institucional que trasciende lo organizacional. En este sentido, las investigaciones analizadas indican que las prácticas contables han incidido, por ejemplo, en la inserción de los mecanismos del mercado en la autoridad local de Nueva Zelanda y sus efectos en la subjetividad de los trabajadores públicos (Ochoki, 2009), en la configuración de centros de costos (codificación de los espacios) en una entidad española durante el siglo XVIII y la visualización de los responsables en la producción de tabaco (Carmona et al., 2002), en la resignificación de los espacios de control en la sociedad actual desde la Ley Sarbanes-Oxley en Estados Unidos (Stein, 2008) y en los sistemas contemporáneos de gestión computarizada en la línea de los impuestos parisinos (Brivot y Gendron, 2011). Además, la pedagogía calculadora ha facilitado distribuir las ayudas humanitarias, como en 
el caso del terremoto de 2009 en la región de Abruzzo en Italia (Sargiacomo, 2015), y estudiar la explotación ganadera dentro de la reserva de Navajos en Estados Unidos (Preston, 2006).

Respecto al eje temático de la modernización del gobierno se identificaron 16 trabajos. En estos se reconoce que el sector público ha sido objeto de una evaluación y renovación de fondo en los últimos 30 años, con un número significativo de países que han implementado las herramientas del NPM y desde allí han adoptado cambios en la contabilidad estatal (Barretta \& Busco, 2011). Por consiguiente, se ha promovido la rendición de cuentas heredada de la doctrina católica (Mutch, 2016), la racionalidad mercantil adaptada para gestionar la cartera de los ciudadanos (Manochin, Brignall, Lowe y Howell, 2011), el discurso del desarrollo sostenible (Birkin, Edwards y Woodward, 2005), la lucha global contra la corrupción (Everett, Neu y Rahaman, 2007), los estándares internacionales de contabilidad (Lehman, 2005; Lima y Craig, 2007; Pelger, 2016; Wall y Connolly, 2016), la privatización del Estado con efectos en las crisis financieras, como por ejemplo en Grecia (Morales, Gendron y Guénin-Paracini, 2014) y los servicios públicos, como el caso del agua en Ghana (Rahaman, Everett y Neu, 2007).

Así, este eje evidencia la interacción entre discursos e instrumentos que tratan de reformar y modernizar el gobierno mediante las prácticas contables desde su dimensión social, institucional y organizacional. Por ejemplo, el análisis del debate sobre la campaña nacional de fitness en el Reino Unido ilustra la manera como las prácticas de cálculo quedaron ligadas a la dieta estatal (Jeacle, 2016). También en el ámbito de la salud, la implementación de algunas reformas desde la NPM muestra como resultado la generación de prácticas organizativas híbridas, por procesos institucionales que surgen de la intervención reguladora y que generan discursos ambiguos como la cooperación y la competencia (Kurunmäki y Miller, 2011a y 2011b). Además, la resistencia de los médicos respecto a la intromisión de las prácticas de la contabilidad en esta profesión (Kurunmäki, 2004), ya que las reformas en este ámbito promueven la creación de "médicos gerentes" que deben controlar los costos de sus propios procesos (Campanale y Cinquini, 2016), en detrimento de la calidad de un servicio público que se debe más a lo social que a lo económico y el desdibujamiento de su ethos profesional.

Finalmente, en el eje temático sobre la contabilidad de la ciudad se identificaron seis trabajos. Este tema cobra especial relevancia para el caso de las ciudades, ya que se observa un doble proceso: por un lado, la visualización de la ciudad, y por el otro, la ciudad calculable desde las presiones de la NPM. Así se pone de manifiesto la importancia de la contabilidad como una tecnología que se inserta en la gestión pública, dada la creciente tendencia de representar las ciudades mediante números y hacerlas comparables desde retóricas cuantitativas y gerenciales en las que la contabilidad ofrece las cifras más relevantes (Lapsley, Miller y Panozzo, 2010). En este eje, el análisis de la gubernamentalidad permite examinar los programas de gobierno para develar concepciones del individuo en tanto consumidor, productor y ciudadano, además de las formas de calcular el direccionamiento y los comportamientos de la gente en la ciudad (McKinlay y Pezet, 2010).

El siglo XXI evidencia una condensación de la población y de las problemáticas en el ámbito urbano. Kornberger y Carter (2010) plantean que el creciente desarrollo de las estrategias en las ciudades se debe a la competitividad; allí vinculan la cuantificación y clasificación de los aspectos de la vida y su rol en el posicionamiento de la ciudad en el escenario global. También, Rautiainen (2010) examina las presiones de la NPM sobre las actividades de control y la medición del desempeño en dos metrópolis finlandesas; concluye que los mecanismos transferidos del sector privado al público han sido demasiado simples para la complejidad de gestión que ellas demandaban.

Por su parte, Maran, Bracci y Funnell (2016), desde un análisis sobre la historia de la contabilidad en una ciudad italiana, advierten sobre la importancia de las prácticas de cálculo y la gestión del poder en el sector público, mientras que Czarniawska (2010) plantea un desafío a los investigadores contables desde la complejidad de la contabilidad de la ciudad, ya que con un ejemplo específico de un proyecto urbano en Roma analiza cómo los políticos manipulan las cuentas y el carácter hermenéutico de las cuentas contables para mostrar la poca transparencia en los procesos reales. 


\section{Principales autores y redes de cooperación}

En el panorama de los registros bibliográficos analizados se identificaron 95 autores. En la Tabla 1 se muestran los cinco autores que más han publicado y sus instituciones de adscripción en las revistas seleccionadas para la revisión del efecto Foucault.

TABLA 1

Autores e instituciones de origen

\begin{tabular}{lc}
\hline Autor (institución) & Número de artículos \\
\hline Dean Neu (University of Calgary / York University) & 8 \\
\hline Cameron Graham (University of Calgary / York University) & 5 \\
\hline Eric Pezet (Université Paris Ouest Nanterre La Défense / CGS, Ecole des Mines) & 4 \\
\hline Ingrid Jeacle (University of Edinburgh) & 4 \\
\hline Peter Miller (London School of Economics and Political Science) & 4 \\
\hline Total & 25 \\
\hline
\end{tabular}

Fuente: elaboración propia.

De los 25 artículos que corresponden a los autores que más han publicado en las revistas seleccionadas, se resalta que el $90 \%$ fue escrito en coautoría, lo cual puede deberse a un trabajo grupal e interdisciplinario para las investigaciones contables y de gestión pública en los estudios en gubernamentalidad. En la totalidad de los artículos, la dinámica de las coautorías se analizó desde las redes de cooperación identificadas entre las 66 universidades de adscripción de los autores y los 16 países a los que pertenecen estas instituciones. Los resultados muestran procesos de cooperación por coautoría entre autores de diferentes universidades y una red de trabajo liderada por Reino Unido (Figura 2), donde, como se señaló en la parte introductoria, se creó el grupo de Historia del presente y se ha avanzado en torno a las discusiones de las necesidades neoliberales de un Estado gerencial. 


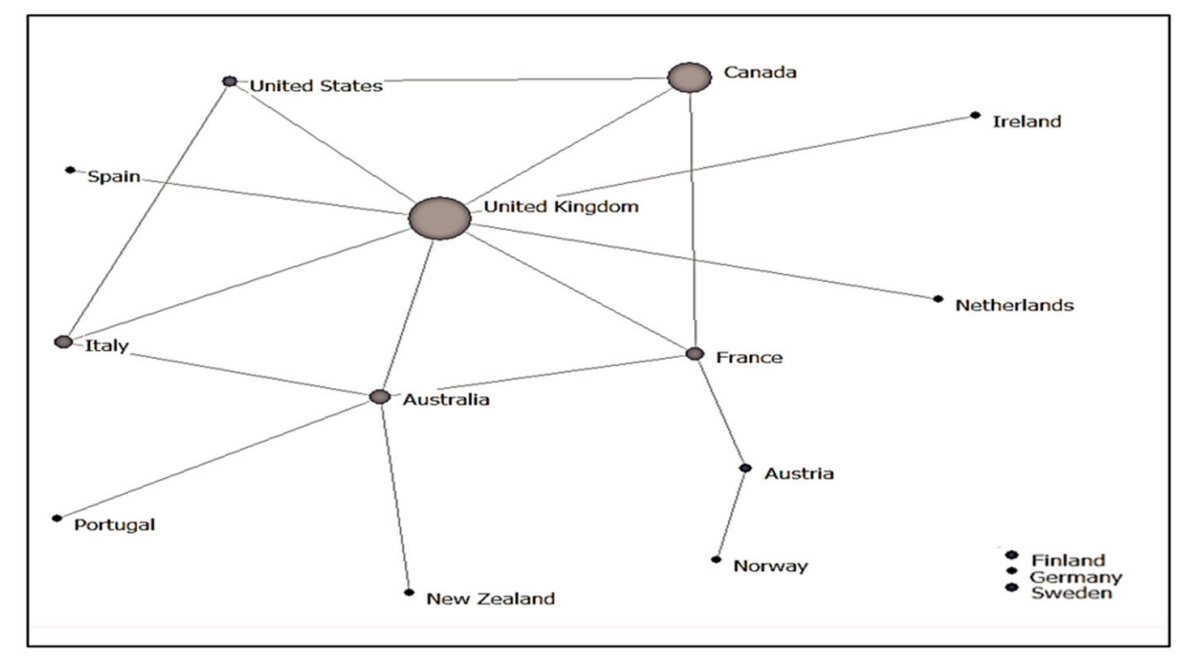

FIGURA 2.

Redes de cooperaciones por países.

Fuente: elaboración propia.

Para el análisis de la red se tuvo presente que los círculos representan a cada país, su tamaño y el nivel de publicación, mientras que las líneas significan la cooperación. En este sentido, la Figura 2 evidencia que en instituciones pertenecientes al Reino Unido (London School of Economics and Political Science, University of St. Andrews y University of Edinburgh) y Canadá (University of Calgary, York University y University of Alberta) se concentraron las publicaciones. Junto con lo anterior se muestra que el Reino Unido ha sido el país que más ha logrado impulsar el efecto Foucault en las investigaciones contables y de gestión pública que fueron analizadas, pues constituye el centro de la red conformada por 13 países, de los cuales seis (Estados Unidos, Canadá, Reino Unido, Italia, Australia y Francia) articulan propiamente la cooperación. También la red presenta una división por autores adscritos a universidades de tres países: Finlandia, Alemania y Suecia, que no escribieron en conjunto con profesores de otras instituciones.

Respecto a lo expuesto se resalta que Foucault ha tenido un lugar privilegiado en el pensamiento inglés, no en vano el evento fundacional de la red de Historia del presente se realizó en Londres. Los británicos Peter Miller y Nikolas Rose delinearon un programa de investigación que se convirtió en la marca de identidad de los estudios gubernamentales desde finales de la década de los años ochenta (Castro-Gómez, 2010), además del profesor inglés Anthony Hopwood, quien al fundar la revista Accounting, Organizations and Society impulsó la difusión del pensamiento contable heterodoxo. En particular, Miller (quien es contable), junto con algunos de sus colegas, como Liisa Kurunmäki y Ted O’Leary, ha promovido el uso de la caja de herramientas conceptuales de Foucault, para entender que las prácticas contables, su cálculo, van de la mano con la conformación de la subjetividad o formas de ser persona. Como lo indica Armstrong (1994) los trabajos más desafiantes en la historia y la sociología de la contabilidad, pese a las críticas de Neimark (1990), se inspiraron en el trabajo del francés Michel Foucault.

\section{Vacíos y retos de investigación}

Las ciudades occidentales del siglo XXI presentan nuevas manifestaciones que deben ser estudiadas desde la visión de los profesionales e investigadores en contaduría y administración. En particular, su extensión planetaria o la urbanización a escala global, el creciente aumento de la población en las ciudades, las prácticas del cálculo contable presentes en muchas dimensiones de la vida cotidiana, la inserción de algunos discursos gerenciales en el gobierno de lo público, además de la fuerza de la academia, detallada en los trabajos 
revisados, sugieren la necesidad de estudios interdisciplinarios respecto a la contabilidad de la ciudad. En este contexto, algunos autores del pensamiento contable heterodoxo han retomado la categoría foucaultiana de gubernamentalidad en sus trabajos, para señalar que las ciudades han recibido poca atención de los investigadores contables y ponen de manifiesto la importancia de estudiar la contabilidad en la gestión pública urbana (Lapsley et al., 2010).

Pese a este llamado, el análisis del efecto Foucault en las investigaciones seleccionadas sobre contabilidad y gestión pública desde la categoría gubernamentalidad permite observar que solo el $9 \%$ de los setenta trabajos revisados corresponde al eje temático: contabilidad de la ciudad; en ese porcentaje, ningún estudio examinó críticamente una ciudad de las llamadas economías emergentes. En general, los estudios seleccionados no hacen evidente las limitaciones de los hallazgos a partir de la crítica al uso de las herramientas conceptuales propuestas por Foucault, pero sí algunos vacíos que permiten plantear la necesidad de trabajos que articulen en los análisis las presiones de tipo de la NPM para modernizar el gobierno de la ciudades (Cristofoli et al., 2010), el campo y las reglas de juego que se definen con las reformas en la contabilidad de la ciudad (Kornberger y Carter, 2010) y los papeles inesperados que, de acuerdo con el contexto, adquiere la contabilidad en la configuración de la vida económica y social urbana (Lapsley et al., 2010), es decir, en el control de los sujetos dentro del aparato estatal y el ajuste de los fenómenos de la población en pro de los objetivos políticos de la administración del ente público.

\section{Discusiones y conclusiones}

Esta investigación muestra unos resultados que permiten ampliar el panorama del efecto que ha tenido Michel Foucault en los estudios de contabilidad y gestión en el ámbito estatal. Quedan en evidencia cuatro ejes que problematizan las temáticas en este tipo de análisis, ellos son: la gestión calculada, el gobierno a distancia, la modernización del gobierno y la contabilidad de la ciudad. También, el detalle de los autores (Neu, Graham, Pezet, Jeacle y Miller) que más publicaron en las revistas seleccionadas respecto al objeto estudiado, además del papel preponderante que tuvo el pensamiento contable inglés en las redes de cooperación, pues Reino Unido fue el país que más impulsó la investigación en contabilidad y gestión pública desde la perspectiva de la gubernamentalidad.

Adicionalmente, el panorama presentado permite resaltar el reto de estudiar la contabilidad en el contexto de la gestión de las ciudades. De los cuatros ejes temáticos ya enunciados, la contabilidad de la ciudad fue el menos investigado. Si bien las ciudades han sido analizadas desde numerosas perspectivas disciplinarias, entre ellas están: la planificación urbana (Jacobs, 1961; 1984), la geografía (Massey, 2005; 2007; Harvey, 2013), la sociología e historia (Mumford, 1938; 1961; Pirenne, 1925; Weber, 1987) y los estudios urbanos y las políticas públicas (Turok y Mykhnenko ,2007), no hay un avance significativo de investigaciones que vinculen lo contable con la ciudad. Se hace énfasis en este problema, porque existen tensiones entre los enfoques disciplinarios del espacio y el diseño con el imperativo financiero de gestión de las ciudades, en un mundo cada vez más dominado por las ideas de la NPM y la primacía de las finanzas (Lapsley et al., 2010). En consecuencia, se propone la ciudad calculable como una de las categorías de análisis, porque es un vacío de investigación desde los estudios en gubernamentalidad.

Si bien en los resultados expuestos se presentan una división por ejes temáticos, por asuntos analíticos, el desarrollo de la categoría propuesta (la ciudad calculable) implicaría su articulación. Así, la contabilidad de la ciudad puede relacionar los otros tres ejes evidenciados en la revisión de literatura, a saber: 1) la gestión calculada; 2) el gobierno a distancia, y 3 ) la modernización del gobierno. El primero, porque una de las métricas y cálculo más relevantes que retoma la doctrina dominante (NPM) resultan de la contabilidad; con ella, las finanzas se reafirman cada vez más como un factor clave ante otras variables para administrar la ciudad. El segundo, dado que en el estudio de la contabilidad de la ciudad se reconoce un cambio de un Estado de 
bienestar (más propio de una sociedad disciplinaria) a un Estado neoliberal (más propio de una sociedad de control). En este último se ejerce más un gobierno a distancia, una acción indirecta en la población, en sus normas y reglas de juego. Y finalmente el tercero, ya que es posible hablar de otra racionalidad política que ha favorecido la inserción de herramientas del NPM para modernizar la administración estatal, con ello el traslado de prácticas contables empresariales que construyen los espacios calculables -físicos y abstractosque los ciudadanos habitan (Mennicken y Miller, 2012).

Ahora bien, revisado el contexto latinoamericano se destacan trabajos realizados sobre la gestión o la contabilidad en clave foucaultiana, por ejemplo, Francisco Ortega (2007), Marcela Zangaro (2011), Naila Flor (2013) y Mauricio Gómez (2013); sin embargo, solo este último autor presenta el NPM en su horizonte analítico. Con lo anterior se plantea que también en el ámbito regional han sido escasos los aportes relacionados con el problema de la contabilidad, la ciudad y los cambios en la gestión estatal desde un análisis foucaultiano. En consecuencia, se amplía el reto de estudiar la contabilidad de la ciudad en un contexto de economía emergente o en proceso de desarrollo. Por ejemplo, en las ciudades de Latinoamérica este vacío posibilitaría analizar la inserción de la NPM en un contexto diferente al de los países del centro de desarrollo en Occidente, mostrar las particularidades de un gobierno neoliberal implementado en áreas urbanas con profundas desigualdades sociales, pero con arraigos culturales muy fuerte del rol protagónico, de la apuesta por los rankings y la visualización de las ciudades como pantalla empresarial urbana. Allí puede ser posible evidenciar el posicionamiento de un discurso de la ciudad competitiva, que cada día adopta los lenguajes y las características de la empresa para la inversión del capital nacional y extranjero, en vez de una ciudad para vivir, en la que el ciudadano se reconozca como tal.

A partir de lo enunciado se proponen retos para avanzar en las reflexiones y comprensiones de la contabilidad en la perspectiva de los estudios en gubernamentalidad en el contexto de las ciudades latinoamericanas. Aquí se presta atención al análisis de un problema que es necesario abordar y discutir en la academia para fortalecer los procesos de cambio en el gobierno de las poblaciones y su incidencia en la construcción de ciudadanos:

Reto 1: identificar las condiciones de posibilidad (económicas, sociales, culturales, políticas, geopolíticas e institucionales) que han permitido la inserción y desarrollo de la NPM y con ello la contabilidad de la ciudad en el contexto latinoamericano.

Reto 2: analizar las relaciones de saber/poder y subjetividad neoliberal propia de las prácticas y discursos contables contemporáneos de la gestión de la ciudad en Latinoamérica.

Reto 3: explicar la racionalidad neoliberal que agencian las prácticas y discursos contables contemporáneos en el gobierno de la ciudad en Latinoamérica.

Reto 4: relacionar las condiciones de aceptabilidad de la población en programas neoliberales que han fundamentado sus cambios en prácticas y discursos de la contabilidad de la ciudad.

Para el desarrollo de estos retos, de sus implicancias y relacionamientos, el artículo ha mencionado diferentes autores que constituyen referencias clave, más no únicas, para avanzar en la comprensión del problema sobre la contabilidad de la ciudad. Y estas referencias no son únicas, porque una limitación, casi natural, de este tipo de artículos, que se fundamentan en una revisión de literatura, es la selección de las revistas objeto de análisis en la que se dejan atrás otras, así como el periodo de estudio determinado. Junto a ello, otra limitación de este trabajo es que la unidad de análisis para el efecto Foucault solo toma la categoría de gubernamentalidad, que obedece a las reflexiones finales de este autor. A esto se suma la clasificación en los cuatro ejes temáticos identificados, que, si bien constituyen un hallazgo, la decisión de clasificación depende del marco de referencia y del proceso de interpretación del investigador. Por ende, se indica que esta propuesta y los retos de investigación son unas posibilidades, entre muchas más otras, que no están exentas de discusión y controversia. 


\section{Agradecimientos}

Este artículo hace parte de la tesis doctoral La contabilidad pública como una tecnología de gubernamentalidad. Un análisis foucaultiano de la gestión del municipio de Medellín (1990-2015), dirigida por el historiador Juan Carlos Jurado Jurado. Agradezco al profesor y a la Universidad Eafit por su apoyo para la realización de esta tesis doctoral en el marco de las becas-crédito de la Dirección de Investigaciones, entre 2012 y 2016.

\section{Referencias bibliográficas}

Anessi-Pessina, E., Barbera, C., Sicilia, M. y Steccolini, I. (2016). Public Sector Budgeting: a European Review of Accounting and Public Management Journals. Accounting, Auditing \& Accountability Journal, 29 (3), 491-519. https://doi.org/10.1108/AAAJ-11-2013-1532.

Armstrong, P. (1994). The Influence of Michel Foucault on Accounting Research. Critical Perspectives on Accounting, 5 (1), 25-55. https://doi.org/10.1006/cpac.1994.1003

Asdal, K. (2011). The office: The Weakness of Numbers and the Production of Non-Authority. Accounting, Organizations and Society, 36 (1), 1-9. https://doi.org/10.1016/j.aos.2011.01.001

Aubert, N., y De Gaulejac, V. (1993). El coste de la excelencia ¿Del caos a la lógica o de la lógica al caos? Barcelona: Ediciones Paidós Ibérica S.A.

Barthes, R. (1999). Mitología. México: Siglo XXI.

Bigoni, M. y Funnell, W. (2015). Ancestors of Governmentality: Accounting and Pastoral Power in the 15th Century. Critical Perspectives on Accounting, 27, 160-176. https://doi.org/10.1016/j.cpa.2014.05.001

Birkin, F., Edwards, P. y Woodward, D. (2005). Accounting's Contribution to a Conscious Cultural Evolution: an end to Sustainable Development. Critical Perspectives on Accounting, 16 (3), 185-208. https://doi.org/10.1016/ S1045-2354(03)00023-6

Brivot, M. y Gendron, Y. (2011). Beyond Panopticism: On the Ramifications of Surveillance in a Contemporary Professional Setting. Accounting, Organizations and Society, 36 (3), 135-155. https://doi.org/10.1016/ j.aos.2011.03.003

Broadbent, J. y Guthrie, J. (2008). Public Sector to Public Services: 20 Years of Contextual Accounting Research. Accounting, Auditing \& Accountability Journal, 21 (2), 129-169. https://doi.org/10.1108/09513570810854383

Brusca, I. (2010). Treinta años de investigación en contabilidad y gestión pública en España. Revista de ContabilidadSpanish Accounting Review, 13(2), 175-209. https://doi.org/10.1016/S1138-4891(10)70016-9

Burchell, G., Gordon, C. y Miller, P. (1991). The Foucault Effect: Studies in Governmentality. With two Lectures by and an Interview with Michel Foucault. Chicago: The University of Chicago.

Burchell, S., Clubb, C., Hopwood, A., Hughes, J. y Nahapiet, J. (1980). The Roles of Accounting in Organizations and Society. Accounting, Organizations and Society, 5 (1), 5-27. https://doi.org/10.1016/0361-3682(80)90017-3

Cabrero, E. (2011). Cambio en la administración pública. En G. Cejudo, Nueva gestión pública, 75-113. México: Siglo XXI editores.

Campanale, C. y Cinquini, L. (2016). Emerging Pathways of Colonization in Healthcare from Participative Approaches to Management Accounting. Critical Perspectives on Accounting, 39, 59-74. https:// doi.org/10.1016/j.cpa.2015.12.001

Carmona, S., Ezzamel, M. y Gutiérrez, F. (2002). The Relationship between Accounting and Spatial Practices in the Factory. Accounting, Organizations and Society, 27 (3), 239-274. https://doi.org/10.1016/ S0361-3682(01)00028-9

Castro, E. (2007). Biopolítica y Gubernamentalidad. Temas \& Matizes (11), 8-18.

Castro-Gómez, S. (2010). Historia de la Gubernamentalidad. Razón de Estado, liberalismo y neoliberalismo en Michel Foucault. Bogotá: Siglo del Hombre Editores. 
Chevallier, J. (2011). El Estado posmoderno. Bogotá: Universidad Externado de Colombia.

Chiapello, E. (2016). Critical Accounting Research and Neoliberalism. Critical Perspectives on Accounting, online. https://doi.org/10.1016/j.cpa.2016.09.002

Chiapello, E. y Baker, C. (2011). The Introduction of French Theory into English Language Accounting Research. Accounting, Auditing \& Accountability Journal, 24 (2), 140-160. https://doi.org/10.1108/09513571111100663

Cîrstea, A. (2014). The Need for Public Sector Consolidated Financial Statements. Procedia Economics and Finance (15), 1289-1296. https://doi.org/10.1016/S2212-5671(14)00590-5

Corvellec, H., Ek, R., Zapata, P. y Zapata, M. J. (2016). Acting on Distances: A Topology of Accounting Inscriptions. Accounting, Organizations and Society, online. https://doi.org/10.1016/j.aos.2016.02.005

Cristofoli, D., Ditillo, A., Liguori, M., Sicilia, M. y Steccolini, I. (2010). Do Environmental and Task Characteristics Matter in the Control of Externalized Local Public Services? Accounting, Auditing \& Accountability Journal, 23 (3), 350-372. https://doi.org/10.1108/09513571011034334

Czarniawska, B. (2010). Translation Impossible? Accounting for a City Project. Auditing \& Accountability Journal, 23 (3), 420-437. https://doi.org/10.1108/09513571011034361

Deleuze, G. (1994). Lógica del sentido. Barcelona: Paidós.

Deleuze, G. (1999). Post-scriptum sobre las sociedades de control. En G. Deleuze, Conversaciones, 277-285. Valencia: Pre-Textos.

Du Gay, P. (2003). Organización de la identidad: gobierno empresarial y gestión pública. En P. Du Gay, Cuestiones de identidad cultural, 251-280. Madrid: Amorrortu.

Du Gay, P. (2012). En elogio de la burocracia. Madrid: Siglo XXI.

Edgley, C. (2010). Backstage in Legal Theatre: a Foucauldian Interpretation of 'Rationes Decidendi' on the Question of Taxable Business Profits. Critical Perspectives on Accounting, 21 (7), 560-572. https://doi.org/10.1016/ j.cpa.2010.03.008

Everett, J., Neu, D. y Rahaman, A. (2007). Accounting and the Global Fight Against Corruption. Accounting, Organizations and Society, 32 (6), 513-542. https://doi.org/10.1016/j.aos.2006.07.002

Ezzamel, M. y Hoskin, K. (2002). Retheorizing Accounting, Writing and Money with Evidence from Mesopotamia and Ancient Egypt. Critical Perspectives on Accounting, 13 (1),333-367. https://doi.org/10.1006/ cpac.2001.0500

Flor, N. (2013). La contabilidad vista como dispositivo de poder: aproximación interpretativa desde la perspectiva foucaultiana. Cuadernos de Contabilidad, 14 (34), 133-158.

Foucault, M. (1994). Hermenéutica del sujeto. Madrid: Las Ediciones de la Piqueta.

Foucault, M. (2001). El sujeto y el poder. En H. Dreyfus, \& P. Rabinow, Michel Foucault: más allá del estructuralismo y la hermenéutica, 241-257. Buenos Aires: Nueva Visión.

Foucault, M. (2008). Nacimiento de la biopolítica. Buenos Aires: Fondo de Cultura Económica.

Foucault, M. (2011). Seguridad, territorio, población. Buenos Aires: Fondo de Cultura Económica.

Giraudeau, M. (2012). Remembering the Future: Entrepreneurship Guidebooks in the US, from Meditation to Method (1945-1975). Foucault Studies, 9 (13), 40-66.

Gordon, C. (1991). Governmental Rationality: An Introduction. En G. Burchell, C. Gordon, \& P. Miller, The Foucault Effect: Studies in Governmentality: with Two Lectures by and an Interview with Michel Foucault, 1-51. Chicago: The University of Chicago Press.

Gómez, M. (2013). Reformas de la contabilidad pública y la construcción del sujeto neoliberal. Recuperado el 03 de mayo de 2016, de https://www.youtube.com/watch?v=WwRcc8FBTUI

Graham, C. (2010). Accounting and the Construction of the Retired Person. Accounting, Organizations and Society, 35 (1), 23-46. https://doi.org/10.1016/j.aos.2009.03.001

Graham, C. (2012). The Subject of Retirement. Foucault Studies (13), 25-39. http://dx.doi.org/10.22439/ fs.v0i13.3505 
Hardt, M. y Negri, A. (2012). Declaración. Madrid: Ediciones Akal S.A.

Harvey, D. (2013). Ciudades rebeldes: del derecho de la ciudad a la revolución urbana. Madrid: Akal.

Helden, J. y Uddin, S. (2016). Public Sector Management Accounting in Emerging Economies: a Literature Review. Critical Perspectives on Accounting, 41, 34-62. https://doi.org/10.1016/j.cpa.2016.01.001

Himick, D. (2009). Accounting and Chilean Pension Reform. Accounting, Auditing \& Accountability Journal, 22 (3), 405-428. https://doi.org/10.1108/09513570910945679

Himick, D. (2015). Human Depreciation Accounting and the Emergence of Industrial Pensions: Linking Human Assets to the Firm. Accounting, Auditing \& Accountability Journal, 242-262. https://doi.org/10.1108/ AAAJ-07-2013-1403

Himick, D. (2016). Actuarialism as Biopolitical and Disciplinary Technique. Accounting, Organizations and Society, 54, 22-44. https://doi.org/10.1016/j.aos.2016.08.005

Hood, C. (1991). A Public Management for All Season? Public Administration, 69 (1), 3-19. https://doi.org/10.1111/ j.1467-9299.1991.tb00779.x

Hood, C. (1995). The "New Public Management" in the 1980s: Variations on a Theme. Accounting, Organizations and Society, 20 (2/3), 93-109. https://doi.org/10.1016/0361-3682(93)E0001-W

Hopwood, A. (1992). Accounting Calculation and the Shifting Sphere of the Economic. European Accounting Review, 1 (1), 125-143. http://dx.doi.org/10.1080/09638189200000007

Hoskin, K. (2015). "What about the box?" Some Thoughts on the Possibility of 'Corruption Prevention', and of 'the Disciplined and Ethical Subject'. Critical Perspectives on Accounting, 28, 71-81. https://doi.org/10.1016/ j.cpa.2015.01.011

Huber, C. y Scheytt, T. (2013). The Dispositif of Risk Management: Reconstructing Risk Management After the Financial Crisis. Management Accounting Research, 24 (2), 88-99. https://doi.org/10.1016/j.mar.2013.04.006

Jacobs, J. (1961). The Death and Life of American Cities. New York: Random House.

Jacobs, J. (1984). Cities and the Wealth of Nations. New York: Random House.

Jeacle, I. (2012). Accounting and Popular Culture: Framing a Research Agenda. Accounting, Auditing \& Accountability Journal, 25 (4), 580-601. https://doi.org/10.1108/09513571211225051

Jeacle, I. (2016). The Diet of the Nation: the State, Family Budgets and the 1930s Nutritional Crisis in Britain. Critical Perspectives on Accounting, 38, 54-68. https://doi.org/10.1016/j.cpa.2015.01.015

Jones, M. (2010). Sources of Power and Infrastructural Conditions in Medieval Governmental Accounting. Accounting, Organizations and Society, 35, 81-94. https://doi.org/10.1016/j.aos.2009.01.005

Jordan, S., Mitterhofer, H. y Jørgensen, L. (2016). The Interdiscursive Appeal of Risk Matrices: Collective Symbols, Flexibility Normalism and the Interplay of 'Risk' And 'Uncertainty'. Accounting, Organizations and Society, online. https://doi.org/10.1016/j.aos.2016.04.003

Kornberger, M. y Carter, C. (2010). Manufacturing Competition: How Accounting Practices Shape Strategy Making in Cities. Accounting, Auditing \& Accountability Journal, 23 (3), 325-349. https:// doi.org/10.1108/09513571011034325

Kurunmäki, L. (2004). A Hybrid Profession-the Acquisition of Management Accounting Expertise By Medical Professionals. Accounting, Organizations and Society, 29 (3-4), 327-347. https://doi.org/10.1016/ S0361-3682(02)00069-7

Kurunmäki, L. y Miller, P. (2011a). Regulatory Hybrids: Partnerships, Budgeting and Modernising. Management Accounting Research, 22 (4), 220-241. https://doi.org/10.1016/j.mar.2010.08.004

Kurunmäki, L. y Miller, P. (2011b). Accounting Within and Beyond The State. Management Accounting Research, 22 (1), 1-5. https://doi.org/10.1016/j.mar.2010.11.003

Lambert, C. (2010). The Making of the Management Accountant-Becoming the Producer. Accounting, Organizations and Society, 36 (1), 10-30. https://doi.org/10.1016/j.aos.2010.07.005

Lambert, C. y Pezet, E. (2012). Accounting and the Making of Homo Liberalis. Foucault Studies, 9 (13), 67-87. http:// dx.doi.org/10.22439/fs.v0i13.3507 
Lapsley, I., Miller, P. y Panozzo, F. (2010). Accounting for the City. Accounting, Auditing \& Accountability Journal, 23 (3), 305-324. https://doi.org/10.1108/09513571011034316

Laval, C. y Dardot, P. (2013). La nueva razón del mundo. Ensayo sobre la sociedad neoliberal. Barcelona: Gedisa S.A. Lazzarato, M. (2013). La fábrica del hombre endeudado. Buenos Aires: Amorrortu.

Legendre, P. (2008). Dominium Mundi. El imperio del Management. Buenos Aires: Amorrortu.

Lehman, C., Annisette, M. y Agyemang, G. (2016). Immigration and Neoliberalism: Three Cases and Counter Accounts. Accounting, Auditing \& Accountability Journal, 29 (1), 43-79. https://doi.org/10.1108/ AAAJ-09-2013-1470

Lehman, G. (2005). A Critical Perspective on the Harmonisation of Accounting in a Globalising World. Critical Perspectives on Accounting, 16 (7), 975-992. https://doi.org/10.1016/j.cpa.2003.06.004

Lima, L. y Craig, R. (2007). Assessing International Accounting Harmonization Using Hegelian Dialectic, Isomorphism and Foucault. Critical Perspectives on Accounting, 18 (6), 739-757. https://doi.org/10.1016/ j.cpa.2006.02.007

Lukka, K. y Vinnari, E. (2014). Domain Theory and Method Theory in Management Accounting Research. Accounting, Auditing \& Accountability Journal, 27 (8), 1308-1338. https://doi.org/10.1108/ AAAJ-03-2013-1265

Mahama, H. y Yu Ming, C. (2009). Currency Options Trading Practices and the Construction and Governance of Operational Risk: a Case Study. Accounting, Auditing and Accountability, 22 (4), 626-660. https:// doi.org/10.1108/09513570910955461

Massaro, M., Dumay, J. y Guthrie, J. (2016). On the Shoulders of Giants: Undertaking a Structured Literature Review in Accounting. Accounting, Auditing \& Accountability Journal, 26 (5), 767-801. https://doi.org/10.1108/ AAAJ-01-2015-1939

Massey, D. (2005). For Space. Newbury Park: Sage.

Massey, D. (2007). World City. Cambridge: Polity Press.

Manochin, M., Brignall, S., Lowe, A. y Howell, C. (2011). Visual Modes of Governmentality: Traffic Lights in a Housing Association. Management Accounting Research, 22 (1), 26-35. https://doi.org/10.1016/ j.mar.2010.10.010

Maran, L., Bracci, E. y Funnell, W. (2016). Accounting and the Management of Power: Napoleon's Occupation of the Commune of Ferrara (1796-1799). Critical Perspectives on Accounting, 34, 60-78. https://doi.org/10.1016/ j.cpa.2015.10.008

Martínez, D. (2011). Beyond Disciplinary Enclosures: Management Control in the Society of Control. Critical Perspectives on Accounting, 22 (2), 200-211. https://doi.org/10.1016/j.cpa.2010.06.016

McKinlay, A. y Pezet, E. (2010). Accounting for Foucault. Critical Perspectives on Accounting, 21 (6), 486-495. https:// doi.org/10.1016/j.cpa.2009.08.006

McKinlay, A., Carter, C., Pezet, E. y Clegg, S. (2010). Using Foucault to Make Strategy. Accounting, Auditing \& Accountability Journal, 23 (8), 1012-1031. https://doi.org/10.1108/09513571011092538

Mehrpouya, A. y Samiolo, R. (2016). Performance Measurement in Global Governance: Ranking and the Politics of Variability. Accounting, Organizations and Society, 55, 12-31. https://doi.org/10.1016/j.aos.2016.09.001

Mennicken, A. y Miller, P. (2012). Accounting, Territorialization and Power. Foucault Studies (13), 4-24. http:// dx.doi.org/10.22439/fs.v0i13.3503

Miller, P. (1994). Accounting as Social and Institutional Practice: An Introduction. En A. Hopwood y P. Miller, Accounting as Social and Institutional Practice, 1-39. Cambridge: Cambridge University Press.

Miller, P. y O'leary, T. (1987). Accounting and The Construction of The Governable Person. Accounting, 12 (3), 235-265. https://doi.org/10.1016/0361-3682(87)90039-0

Miller, P. y Rose, N. (1990). Governing Economic Life. Economy and Society, 19 (1), 1-31. http:// dx.doi.org/10.1080/03085149000000001 
Morales, J., Gendron, Y. y Guénin-Paracini, H. (2014). State Privatization and the Unrelenting Expansion of Neoliberalism: the Case of the Greek Financial Crisis. Critical Perspectives on Accounting, 25 (6), 423-445. https://doi.org/10.1016/j.cpa.2013.08.007

Mumford, L. (1938). The Culture of Cities. New York: Harcourt Brace.

Mumford, L. (1961). The City in History: Its Origins, Its Transformations, and Its Prospects. London: Secker and Warburg.

Mutch, A. (2016). Religion and Accounting Texts in Eighteenth Century Scotland Organizational Practices and a Culture of Accountability. Accounting, Auditing \& Accountability Journal, 29 (6), 926-946. https:// doi.org/10.1108/AAAJ-07-2014-1753

Napier, C. (2006). Accounts of Change: 30 Years of Historical Accounting Research. Accounting, Organizations and Society, 31 (4-5), 445-507. https://doi.org/10.1016/j.aos.2005.12.004

Neimark, M. (1990). The King is Dead. Long Live the King! Critical Perspectives on Accounting, 1 (1), 443-114. https:// doi.org/10.1016/1045-2354(90)01010-3

Neu, D. (2006). Accounting for Public Space. Accounting, Organizations and Society, 31 (4-5), 391-414. https:// doi.org/10.1016/j.aos.2005.03.001

Neu, D. y Graham, C. (2004). Accounting and the Holocausts of Modernity. Accounting, Auditing \& Accountability Journal, 17 (4), 578-603. https://doi.org/10.1108/09513570410554560

Neu, D. y Graham, C. (2006). The Birth of a Nation: Accounting and Canada's First Nations, 1860-1900. Accounting, Organizations and Society, 31 (1), 47-76. https://doi.org/10.1016/j.aos.2004.10.002

Neu, D., Everett, J. y Rahaman, A. (2015). Preventing Corruption Within Government Procurement: Constructing the Disciplined and Ethical Subject. Critical Perspectives on Accounting, 28, 49-61. https://doi.org/10.1016/ j.cpa.2014.03.012

Neu, D., Ocampo, E., Graham, C. y Heincke, M. (2006). "Informing” Technologies and the World Bank. Accounting, Organizations and Society, 31 (7), 635-662. https://doi.org/10.1016/j.aos.2005.07.002

Ochoki, R. (2009). Construction and Effects of Markets in a Local Authority in New Zealand. Accounting, Auditing \& Accountability Journal, 22 (7), 1055-1086. https://doi.org/10.1108/09513570910987376

O'Connor, J. (2009). The Fiscal Crisis of the State. New Jersey: Transaction Publishers.

Offe, C. (1991). Contradicciones del Estado del Bienestar. México: Alianza.

Ortega, F. (2007). La contabilidad como mecanismo anátomo y bio-político del poder. Porikan, 9 (12), 179-204.

Osborne, D. y Gaebler, T. (1994). La reinvención del gobierno. La influencia del espiritu empresarial en el sector público. Buenos Aires: Editorial Paidós.

O'Regan, P. (2010). 'A Dense Mass of Petty Accountability': Accounting in the Service of Cultural Imperialism During the Irish Famine, 1846-1847. Accounting, Organizations and Society, 35 (4), 416-430. https://doi.org/10.1016/ j.aos.2009.10.001

Pelger, C. (2016). Practices of Standard-Setting e an Analysis of the IASB's and FASB's Process of Identifying the Objective of Financial Reporting. Accounting, Organizations and Society, 50 (C), 51-73. https:// doi.org/10.1111/j.1467-6281.2005.00182.x

Pirenne, H. (1925). Mediaeval Cities: Their Origins and the Revival of Trade. Princeton: Princeton University.

Preston, A. (2006). Enabling, Enacting and Maintaining Action at a Distance: an Historical Case Study of the Role of Accounts in the Reduction of the Navajo Herds. Accounting, Organizations and Society, 31 (6), 559-578. https:// doi.org/10.1016/j.aos.2005.03.003

Rahaman, A., Everett, J. y Neu, D. (2007). Accounting and the Move to Privatize Water Services in Africa. Accounting, Auditing \& Accountability Journal, 20 (5), 637-670. https://doi.org/10.1108/09513570710778992

Rautiainen, A. (2010). Contending Legitimations. Performance Measurement Coupling and Decoupling in Two Finnish Cities. Accounting, Auditing \& Accountability Journal, 23 (3), 373-391. https:// doi.org/10.1108/09513571011034343 
Rivera, E. (2012). Foucault: His Influence Over Accounting and Management Research. Building of a Map of Foucault's Approach. International Journal of Critical Accounting, 4 (5/6), 728-756. https://doi.org/10.1504/ IJCA.2012.051466

Rose, N. y Miller, P. (2008). Governing the Present: Administering Economic, Social and Personal Life. Cambridge: Polity Press.

Sargiacomo, M. (2015). Earthquakes, Exceptional Government and Extraordinary Accounting. Accounting, Organizations and Society, 42 (1), 67-89. https://doi.org/10.1016/j.aos.2015.02.001

Servalli, S. (2013). The Interface of Power and Charity in the Government of Poor: a Case From The Italian Context in the Sixteenth-Seventeenth Centuries. Accounting, Auditing \& Accountability Journal, 26 (8), 1306-1341. https://doi.org/10.1108/AAAJ-05-2013-1360

Stein, M. (2008). Beyond the Boardroom: Governmental Perspectives on Corporate Governance. Accounting, Auditing \& Accountability Journal, 21 (7), 1001-1025. https://doi.org/10.1108/09513570810907456

Thomson, I., Dey, C. y Russell, S. (2015). Activism, Arenas and Accounts in Conflicts Over Tobacco Control. Accounting, Auditing \& Accountability Journal, 28 (5), 809-845. https://doi.org/10.1108/AAAJ-08-2013-1439

Thomson, I., Grubnic, S. y Georgakopoulos, G. (2014). Exploring Accounting-Sustainability Hybridisation in the UK Public Sector. Accounting, Organizations and Society, 453-476. https://doi.org/10.1016/j.aos.2014.02.003

Tregidga, H. (2013). Biodiversity Offsetting: Problematisation of an Emerging Governance regime. Accounting, Auditing \& Accountability Journal, 26 (5), 806-832. https://doi.org/10.1108/AAAJ-02-2013-1234

Turok, I. y Mykhnenko, V. (2007). The Trajectories of European Cities, 1960-2005. Cities, 24 (3), 165-182. https:// doi.org/10.1016/j.cities.2007.01.007

Vaivio, J. (2006). The Accounting of "The Meeting": Examining Calculability within a "Fluid" Local Space. Accounting, Organizations and Society (31), 735-762. https://doi.org/10.1016/j.aos.2005.12.007

Viana de Souza, F., Corrêa da Silva, M. y Oliveira, A. (2013). Uma análise da produção científica da área de contabilidade governamental nos periódicos que utilizam o SEER e o SciELO. Revista de Contabilidade da UFBA, 7 (2), 22-37.

Walker, S. (2010). Child Accounting and 'the Handling of Human Souls'. Accounting, Organizations and Society, 35 (6), 628-657. https://doi.org/10.1016/j.aos.2010.07.001

Walker, S. (2016). Revisiting the Roles of Accounting in Society. Accounting, Organizations and Society, 49 (1), 41-50. https://doi.org/10.1016/j.aos.2015.11.007

Wall, A. y Connolly, C. (2016). Implementing IFRSs in the UK Devolved Administrations. Accounting, Auditing \& Accountability Journal, 29 (2), 198-225. https://doi.org/10.1108/AAAJ-07-2014-1780

Watkins, A. y Arrington, E. (2007). Accounting, New Public Management and American Politics: Theoretical Insights into the National Performance Review. Critical Perspectives on Accounting, 18 (1), 33-58. https:// doi.org/10.1016/j.cpa.2005.02.003

Weber, M. (1987). La ciudad. Madrid: Las Ediciones de la Piqueta.

Zangaro, M. (2011). Subjetividad y trabajo. Una lectura foucaultiana del management. Buenos Aires: Herramienta.

\section{Notas}

[1] En el artículo Public sector management accounting in emerging economies: Literature Review, los autores relacionan la categoría Governmentality (Foucault) / Bourdieusian framework. Sin embargo, de un total de 69 textos relevados, en esa perspectiva solo se identifican cinco.

\section{Licencia Creative Commons CC BY 4.0}

Para citar este artículo: Ocampo-Salazar, C. A. (2018). Un panorama del efecto Foucault en contabilidad y gestión pública. Temáticas, autores y retos de investigación desde la gubernamentalidad. Cuadernos de Contabilidad, 19(47), 60-79. https://doi.org/10.11144/Javeriana.cc19-47.pefc 